\title{
«Es lohnt sich!»
}

Interview: Stefan Kühnis

Freischaffender Journalist BR

Wird ein Patient über längere Zeit arbeitsunfähig, sind vor allem die Ärzte gefordert - und zwar nicht nur mit ihrem medizinischen Know-how, sondern auch zugunsten einer möglichst raschen Wiedereingliederung am Arbeitsplatz. Das setzt eine verstärkte Zusammenarbeit voraus. Darüber sprach Stefan Kühnis mit den Allgemeinmedizinern Gabriela Kissling-Winiger und Michael Fluri.

Für alle Beteiligten ist es wichtig, dass kranke oder verunfallte Arbeitnehmer so rasch wie möglich wieder arbeiten können. Denn mit zunehmender Dauer der Arbeitsunfähigkeit nehmen die Chancen einer erfolgreichen Wiedereingliederung ab - nach sechs Monaten bereits um rund 50 Prozent. Woran genau liegt das? Gabriela Kissling-Winiger: Oft liegt es in der Natur der Sache. Manchmal gibt es Verletzungs- oder Krankheitsmuster, die eine schwierige Prognose haben. Andere Male ist es sozioökonomisch oder psychosozial eine schwierige Konstellation. Vor allem dann, wenn ein Arbeitnehmer eher ungebildet oder die Arbeitsplatzzufriedenheit tief ist. Obwohl wir uns der Problematik bewusst sind, können wir den Verlauf nur schwer beeinflussen. Das hört man nicht gerne, es ist aber so. Und sich in diesen Fällen gegenseitig den Schwarzen Peter zuzuschieben, braucht viele Ressourcen und frustriert letztlich alle Beteiligten.

\section{Welche Herausforderungen stellt eine Arbeits- unfähigkeit an die behandelnden Ärzte?}

Michael Fluri: Einerseits geht es um die Objektivierbarkeit. Man muss sich auf die Schilderungen der Beschwerden des Patienten abstützen. Diese können natürlich sehr subjektiv sein. Wie schlecht es jemandem geht und ob man so arbeiten kann oder nicht, das hängt besonders bei Schmerzstörungen oder Stimmungsproblemen stark davon ab, wie sich der Patient fühlt. Es lässt sich nicht oder nur mit sehr aufwendigen Verfahren objektivieren. Eine zweite Herausforderung sind die Kenntnisse über den Arbeitsplatz.

\section{Oft fehlen den Ärzten diese Kenntnisse über} die konkreten Arbeitsbelastungen der Patienten. Wie lässt sich das ändern?

Michael Fluri: Das geht vor allem über das Befragen des Patienten. Die meisten wollen das ja richtig darstellen. Hier muss man dann differenzieren: mit einem Tennisellbogen an der Kasse eines Supermarkts zu

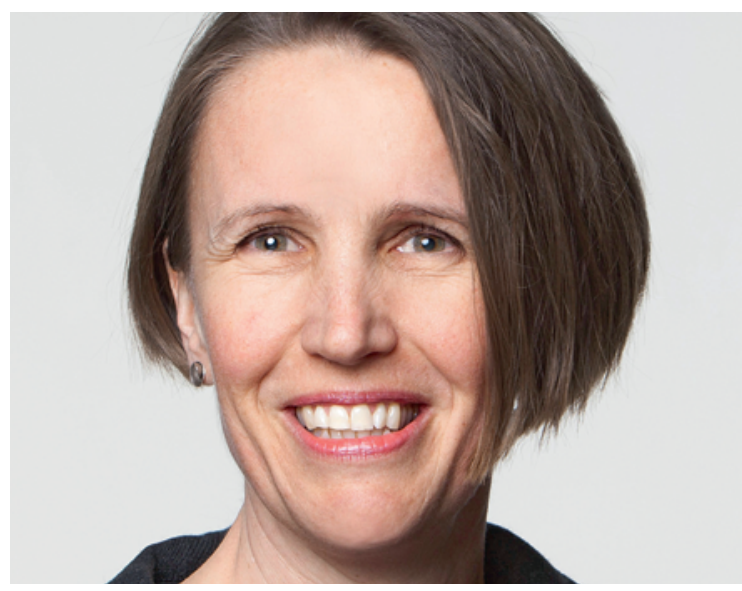

Dr. med. Gabriela Kissling-Winiger ist Co-Präsidentin der Hausärzte Solothurn und Fachärztin für Allgemeinmedizin in der Gruppenpraxis Ziegelfeld in Olten.

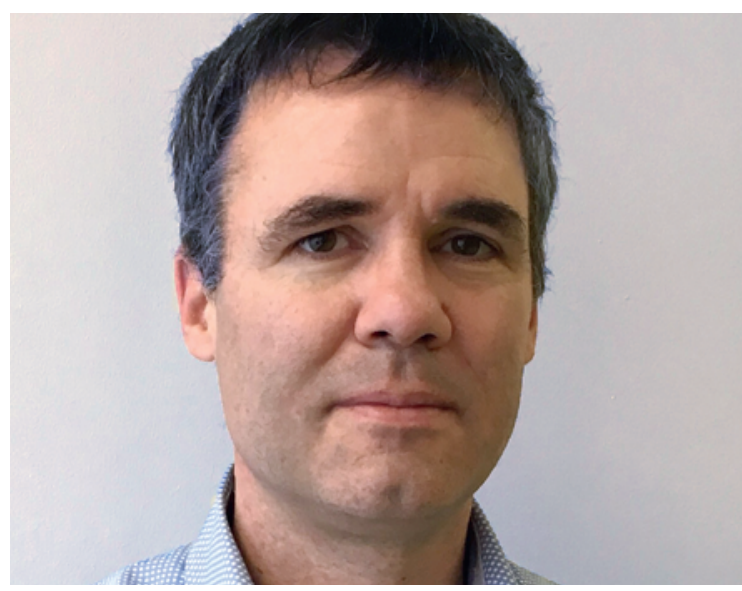

Dr. med. Michael Fluri ist Facharzt für Allgemeine Innere Medizin und für Manuelle Medizin SAMM in der Hausarztpraxis Weissenstein in Langendorf.

arbeiten, geht fast nicht mehr. Als Forstwart in der Kabine einer schweren Maschine geht das jedoch gut, auch wenn das auf den ersten Blick nicht so erscheint. In komplizierteren Fällen kann uns der Arbeitgeber einen Arbeitsplatzbeschrieb zukommen lassen. Dann können wir differenziert Stellung nehmen. 
Gabriela Kissling-Winiger: Meiner Erfahrung nach muss man etwas lockerer den Telefonhörer in die Finger nehmen, am besten in Anwesenheit der Patienten. Wenn wir rasch den Kontakt zum Arbeitgeber herstellen können, lässt es sich am einfachsten beurteilen, was in welchem Rahmen noch geleistet werden könnte. Ansonsten schreibe ich häufig ein Teilarbeitsfähigkeitszeugnis und sage dem Patienten, er solle mit dem Arbeitgeber klären, wofür er ihn einsetzen könne. In der Krankenakte notiere ich den Namen des Vorgesetzten und lasse mir die Entbindung von der Schweigepflicht vom Patienten geben. Dann kann der Patient den Vorgesetzten auffordern, bei Fragen oder Anliegen mit mir Kontakt aufzunehmen. Da der Patient in der Regel auch an einer reibungslosen Zusammenarbeit interessiert ist, klappt das meistens relativ gut. Auch spürt man so schnell, ob ein vertrauensvolles Verhältnis zum Vorgesetzten besteht. Und das ist für den weiteren Verlauf eminent wichtig.

Michael Fluri: Das Telefon in die Hand zu nehmen ist eine gute Möglichkeit, dann hört der Patient auch gleich, was man bespricht. Manchmal ist der Zeitdruck zu gross, um zu telefonieren. Vor allem, wenn man in einem grossen Betrieb erst die richtige Ansprechperson finden muss. Dann darf mich der Arbeitgeber auch gerne anrufen. In meiner Praxis gibt es die klare Devise, dass Arbeitgeber immer direkt zu mir durchgestellt werden, wenn sie anrufen.

\section{Was genau soll und darf eigentlich auf ein Arbeits- unfähigkeitszeugnis?}

Gabriela Kissling-Winiger: Datum, Name, eventuell die Adresse oder das Geburtsdatum, so dass der Patient klar identifiziert werden kann. Hinzu kommen das Datum des Beginns der Arbeitsunfähigkeit und unbedingt ein bestimmter Endtermin oder der Termin der nächsten Beurteilung. Dann braucht es noch die Unterscheidung, ob es eine Krankheit oder ein Unfall ist, und es braucht eine Unterschrift. Alles Weitere darf nicht darauf.

\section{Schulterschluss für eine rasche Wiedereingliederung}

Der Kanton Solothurn macht es vor: Wirtschaftsverbände, Ärzteschaften, die IV-Stelle Solothurn sowie die Suva-Agentur Solothurn setzen sich gemeinsam dafür ein, dass arbeitsunfähige Menschen möglichst rasch an ihren Arbeitsplatz zurückkehren können. Für eine effiziente Umsetzung haben sie Grundsätze und ein entsprechendes Merkblatt erarbeitet. Mit ihrer Unterschrift verpflichten sie sich, diese Grundsätze bei der Zusammenarbeit einzuhalten und so die rasche und gesicherte Wiedereingliederung von Erkrankten oder Verunfallten in den Arbeitsprozess zu fördern.

Der Schulterschluss wird als eines von 10 "good practice»-Beispielen im Rahmen der nationalen Konferenz für die Arbeitsmarktintegration von Menschen mit Behinderung vorgestellt. Infos: www.bsv.admin.ch/bsv/de/home/sozialversicherungen/iv/grundlagen-gesetze/arbeitsmarktintegration/nationale-konferenz/konferenz-i.html

Weitere Informationen:

http://www.ivso.ch/dynamic/page.asp?seiid=59

https://www.suva.ch/de-ch/die-suva/news-und-medien/medien\#uxlibrary-open=/de-CH?

atomid $=\mathrm{d} 4 \mathrm{f} 5 \mathrm{bbcd} 49 \mathrm{e} 24551 \mathrm{~b} 44156 \mathrm{~b} 012 \mathrm{f} 7 \mathrm{fc} 2 \mathrm{~d} \% 26$ showContainer $=1$

Michael Fluri: Unlesbare Zeugnisse oder widersprüchliche Daten gehen auch gar nicht. Es ist schade, wenn Akademiker es nicht zustande bringen, ein solches Zettelchen korrekt und lesbar auszufüllen.

\section{Das heisst aber, ein Austausch mit dem Arbeitgeber} setzt immer das Einverständnis des Patienten voraus? Gabriela Kissling-Winiger: Wenn uns das der Patient nicht erlaubt, dürfen wir den Arbeitgeber nicht kontaktieren. Und auch dann dürfen wir nur das erwähnen, was der Arbeitgeber aus der Krankheitsgeschichte wissen muss, um einen möglichen Einsatz am Arbeitsplatz beurteilen zu können. Das alles geht jedoch nur mit einer dokumentierten Entbindung von der Schweigepflicht.

\section{Was haben Ärzte denn von diesem Dialog?}

Michael Fluri: Meistens ist es eher ein Monolog des Arbeitgebers. Es bringt mir aber sehr viel, wenn ich die Arbeitsbelastungen beschrieben erhalte. So kann ich einfacher weitere Beurteilungen treffen, welche Einschränkungen der Patient für diese Arbeit hat und wie lange das dauern wird.

Gabriela Kissling-Winiger: Wir haben zudem einen viel einfacheren Job, wenn der Arbeitsplatz erhalten bleibt. Es gibt für das ganze Gesundheitswesen keine schlimmere Situation als ein gekündigtes Arbeitsverhältnis und eine gescheiterte Wiedereingliederung. Dann werden Krankheiten chronisch, was hohe Kosten verursacht und auch für uns Ärzte sehr unbefriedigend ist. Juristen werden eingeschaltet, es gibt Begutachtungen, und oft wissen wir schon zu Beginn, dass es keine Rente geben wird. Das alles ist sehr frustrierend. Es ist deshalb unser ureigenes Interesse, diesen Austausch mit den Arbeitgebern zu pflegen. Es lohnt sich!

\section{Weshalb wehren sich manche Ärzte dagegen?}

Michael Fluri: Einige fühlen sich in ihrer Kompetenz angegriffen. Aber das ist falsch. Die Ärzte können viel profitieren, auch dadurch, dass die Arbeitgeber aufgeklärt werden, wie relevant ein solches Arbeitsunfähigkeitszeugnis ist, oder wenn sie wissen, wie es zustande kommt und dass wir nun mal an die Schweigepflicht gebunden sind. Auch die Angst, dass Arbeitgeber die Ärzte bezüglich der Schweigepflicht in Bedrängnis bringen wollen, ist sicher ein Faktor. Und man muss sich daran gewöhnen, dass man als Arzt mehr in Frage gestellt wird. Das ist aber nicht nur schlecht, dadurch kann man eigentlich nur gewinnen. Und wenn die Arbeitgeber hören, dass auch wir der Meinung sind, dass man schnell wieder arbeiten sollte, ist das sicher gut. Ich finde es sehr schade, wenn man sich diese Gelegenheiten zur Zusammenarbeit entgehen lässt. 